\title{
Auxin-cytokinin interactions in the control of shoot branching
}

\author{
Sae Shimizu-Sato $\cdot$ Mina Tanaka $\cdot$ Hitoshi Mori
}

Received: 22 September 2008/ Accepted: 12 October 2008/Published online: 30 October 2008

(C) The Author(s) 2008. This article is published with open access at Springerlink.com

\begin{abstract}
In many plant species, the intact main shoot apex grows predominantly and axillary bud outgrowth is inhibited. This phenomenon is called apical dominance, and has been analyzed for over 70 years. Decapitation of the shoot apex releases the axillary buds from their dormancy and they begin to grow out. Auxin derived from an intact shoot apex suppresses axillary bud outgrowth, whereas cytokinin induced by decapitation of the shoot apex stimulates axillary bud outgrowth. Here we describe the molecular mechanisms of the interactions between auxin and cytokinin in the control of shoot branching.
\end{abstract}

Keywords Adenosine phosphate-isopentenyltransferase Apical dominance $\cdot$ Basipetal auxin flow

Cytokinin oxidase $\cdot$ Pea $\cdot$ PINI $\cdot$ Shoot branching

$\begin{array}{ll}\text { Abbreviations } \\ \text { CK } & \text { Cytokinin } \\ \text { CKX } & \text { Cytokinin oxidase } \\ \text { GUS } & \beta \text {-glucuronidase } \\ \text { IAA } & \text { Indole-3-acetic acid } \\ \text { IPT } & \text { Adenosine phosphate-isopentenyltransferase } \\ \text { SAM } & \text { Shoot apical meristem } \\ \text { TIBA } & 2,3,5 \text {-triiodobenzoic acid }\end{array}$

\section{Introduction}

Apical dominance is a phenomenon in which a main shoot in an intact plant grows predominantly while suppressing

S. Shimizu-Sato $\cdot$ M. Tanaka $\cdot$ H. Mori $(\bowtie)$

Graduate School of Bioagricultural Sciences, Nagoya

University, Chikusa, Nagoya 464-8601, Japan

e-mail: morihito@agr.nagoya-u.ac.jp the outgrowth of axillary buds. After loss of the main shoot, the dormant axillary buds immediately begin to grow out as a main shoot to replace the lost apex, thereby allowing the plant to survive (Cline 1991; Shimizu-Sato and Mori 2001). A main shoot derives from the activity of the primary shoot apical meristem (SAM), which arises during embryogenesis (Kerstetter and Hake 1997). Axillary buds also derive from the primary SAM in a developmental process that generally involves two phases. (1) The axillary meristem is formed from groups of meristematic cells, which originate directly from detached parts of the primary SAM of the main shoot. The axillary meristem produces axillary buds located on the axil of the leaf primordia. (2) After axillary buds are fully developed and have reached a certain size depending on the plant species, growth ceases and the axillary bud becomes dormant. In Arabidopsis, pea, petunia, and tomato, some mutants show a phenotype that is characteristic of a specific developmental phase of the axillary buds. For example, the lateral suppressor ( $l s$ ) mutant in tomato shows decreased branching, because the $l s$ mutation prevents the initiation of axillary meristems during the vegetative phase (Malayer and Guard 1964). The more axillary growth ( $\max$ ) mutant of Arabidopsis shows enhanced shoot branching (Stirnberg et al. 2002), caused by the release of dormant axillary buds.

Shoot branching has an important role in generating a large variety of diverse plant forms, because the degree of apical dominance varies depending on the plant species. Branching pattern complexity also depends on plant age. Generally, older plants show weaker apical dominance resulting in a bushier form in which the total number of leaves or flowers are increased to be more fruitful. Shoot branching is controlled by environmental cues (such as light strength and nutrition) as well as by temporal and spatial developmental programs (Cline 1996). In some 
species, axillary buds of plants damaged by excessive bending continue to grow in the intact shoot apex, suggesting that gravity is also one of the environmental cues that controls axillary bud outgrowth. Interestingly, gravityregulated release from apical dominance seems to be distinct and unique from decapitation-regulated release from apical dominance (Kitazawa et al. 2008). When the upper part of the main shoot of the Japanese morning glory (Pharbitis nil) is bent down, the axillary buds located on the uppermost node of the bent region begin to grow. Despite this phenomenon, shoot bending does not induce significant changes in either the cytokinin (CK) level or the auxin response, which are typical responses to the release from apical dominance caused by decapitation. The response to gravity, therefore, might be a separate molecular mechanism for survival.

Apical dominance is regulated by two plant hormones, auxin and CK. In this review, we describe the molecular mechanisms underlying the interactions between auxin and $\mathrm{CK}$ in the control of shoot branching. Recently, studies of a series of recessive mutants with enhanced shoot branching revealed that a third novel hormone is also involved in inhibiting the outgrowth of axillary buds. These mutants include the pea ramosus (rms) mutant, the petunia decreased apical dominance ( $d a d)$ mutant, the Arabidopsis more axillary growth (max) mutant, and the rice dwarf (d) and high-tillering dwarf (htd) mutants (Beveridge et al. 1994; Napoli 1996; Stirnberg et al. 2002; Ishikawa et al. 2005). Dun et al. and Ongaro and Leyser have provided excellent reviews of other important signals involved in shoot branching (Dun et al. 2006; Ongaro and Leyser 2008).

\section{The basipetal auxin flow in the stem suppresses axillary bud outgrowth}

More than 70 years ago, Thimann et al. reported that decapitation of Vicia spp. plants induces axillary bud outgrowth, whereas the application of indole-3-acetic acid (IAA) to the stump prevents axillary bud outgrowth (Thimann and Skoog 1934). Axillary bud growth cannot be prevented by the direct application of auxin to the axillary buds after decapitation. Further, radiolabeled auxin applied to the stump is not transported into the axillary buds (Hall and Hillman 1975). These findings indicate that auxin does not directly prevent axillary bud outgrowth. Stump application of 2, 4-dichlorophenoxyacetic acid, which cannot be transported basipetally in plants, also does not prevent the outgrowth of axillary buds (Brown et al. 1979). The auxintransport inhibitor 2, 3, 5-triiodobenzoic acid (TIBA) in lanolin applied to the stem of an intact plant can, however, reduce or abolish apical dominance (Snyder 1949). These observations indicate that basipetal auxin flow derived from a shoot apex inhibits axillary bud outgrowth.

Auxin, produced mainly in young leaves and in the shoot apex (Ljung et al. 2001), is transported basipetally down the stem in a polar manner by active transport in the vascular parenchyma (Lomax et al. 1995). Several proteins regulate auxin transport, such as the auxin efflux carriers PIN-FORMED (PIN) and p-glycoprotein, and the auxin influx facilitators AUXIN RESISTANT 1 (AUX1)/LIKEAUX1 (LAX) (Okada et al. 1991; Marchant et al. 1999; Murphy et al. 2002). Although the molecular mechanisms of active auxin transport are becoming clear, it remains unknown how auxin flow derived from the shoot apex induces axillary buds to enter dormancy.

\section{CK promotes axillary bud outgrowth}

The effects of CK in apical dominance are antagonistic to those of auxin. Direct application of CK to axillary buds promotes axillary bud outgrowth, even in intact plants (Wickson and Thimann 1958). To date, CK is the only chemical known to release axillary buds from dormancy. After decapitation in chickpea, CK levels in the axillary buds increase 7-fold within $6 \mathrm{~h}$ and 25-fold within $24 \mathrm{~h}$, and axillary bud outgrowth is well correlated with the CK level in the axillary buds (Turnbull et al. 1997). Roots are the main CK-producing organ in plants, and CK biosynthesized in the root system is transported into the shoot system via the xylem (Hopkins and Huner 2004). CK levels in bean xylem exudates increase within $16 \mathrm{~h}$ after decapitation and gradually return to basal levels (Bangerth 1994). Application of 1-naphthylacetic acid to the stump prevents the increase in $\mathrm{CK}$ in the bean xylem exudates ( $\mathrm{Li}$ et al. 1995). These observations led to the suggestion that CK derived from roots promotes axillary bud outgrowth after decapitation, and auxin derived from a shoot apex regulates CK transport in plants (Letham 1994). It is now known, however, that the $\mathrm{CK}$ that promotes axillary bud outgrowth after decapitation is locally biosynthesized in the nodal stem rather than in the roots (Tanaka et al. 2006), as described below.

\section{An adenosine phosphate-isopentenyltransferase (IPT) gene is expressed specifically in nodal stems after decapitation}

Several molecular approaches have been used to characterize the biochemical events associated with axillary bud dormancy and outgrowth. In pea (Pisum sativum L. cv. Alaska) axillary buds, the molecular mechanisms of cell cycle control during the dormancy-to-growth transition 
were characterized based on the expression patterns of cell cycle regulators such as histone $H 4$ (S phase marker), cyclinB (G2/M phase marker), cyclinD (G1 phase marker), and proliferating cell nuclear antigen (G1/S phase marker) (Shimizu and Mori 1998). These analyses demonstrated that for every $2-\mathrm{cm}$ increase in the distance from the site of decapitation to the second node, there is a 2-h delay in the induction of proliferating cell nuclear antigen mRNA in the axillary buds (Shimizu-Sato et al. unpublished data). This finding suggests that sustained outgrowth of the axillary bud is regulated by a decrease in the auxin level in the stem around the second node, although the rapid but transient initial growth of the axillary bud is dependent on hydrostatic pressure (McIntyre and Damson 1988) and independent of auxin (Morris et al. 2005). The genes, including IPT, expressed in nodal stems after decapitation were identified by a subtraction method using mRNA prepared from nodal stems before or after decapitation (Tanaka et al. 2006). IPT encodes a key enzyme in CK biosynthesis (Kakimoto 2001; Takei et al. 2001). Transgenic Agrobacterium tumefaciens plants overexpressing IPT exhibit elevated levels of $\mathrm{CK}$, and reduced apical dominance (Medford et al. 1989). The petunia mutant sho, which was identified by activation tagging, also has increased levels of $\mathrm{CK}$, and reduced apical dominance (Zubko et al. 2002). sho encodes an IPT protein. These observations indicate that IPT has an important role in the control of CK levels in plants. IPT cDNA has been identified and characterized in various plants such as Arabidopsis, rice, and maize (Kakimoto 2001; Takei et al. 2001). In pea, two full-length IPT cDNAs (PsIPT1 and PSIPT2) were isolated from the stem. PsIPT2 in the stem seems to have a main role in controlling axillary bud outgrowth after decapitation, because PSIPT2 was the only isolated clone identified from a subtracted library prepared from nodal stems after decapitation.

\section{Auxin derived from a shoot apex suppresses local biosynthesis of $\mathrm{CK}$ in the nodal stem through the regulation of PSIPT expression}

Members of the IPT family regulate CK levels in Arabidopsis in response to plant developmental processes (Miyawaki et al. 2004; Takei et al. 2004). In pea plants, PSIPT2 mRNA expressed transiently in the nodal stems increases at $1 \mathrm{~h}$, reaches a maximum level at $3 \mathrm{~h}$, decreases by $9 \mathrm{~h}$, and is undetectable $24 \mathrm{~h}$ after decapitation. This expression pattern suggests that the induction of PSIPT2 mRNA in the nodal stem correlates with the release of dormant axillary buds, and that PsIPT2 is involved in CK biosynthesis to stimulate axillary bud outgrowth. Together, these results and the report that basipetal auxin transport in the pea stem is $1 \mathrm{~cm} \mathrm{~h}^{-1}$ (Johnson and Morris 1989) suggest that auxin derived from the shoot apex represses PSIPT2 expression in the nodal stem in intact plants, and that the depletion of auxin in the nodal stem immediately induces the expression of PSIPT2 mRNA after decapitation (Tanaka et al. 2006).

Some members of the Arabidopsis IPT family are downregulated by $\mathrm{CK}$, and others are upregulated by auxin (Miyawaki et al. 2004). PSIPT2, however, is downregulated by auxin, as demonstrated by the following two experiments. (1) Decrease of PSIPT2 expression by exogenous IAA in nodal excised stem segments, in which PSIPT2 mRNA had accumulated. Three hours after decapitation $1 \mathrm{~cm}$ above the second node, nodal stem segments in which PSIPT2 mRNA was increased were excised. The excised nodal segments were incubated in 2-(N-morpholine)-ethanesulfonic acid (MES) buffer with or without IAA. Northern blot analyses showed that PSIPT2 mRNA was maintained at the same level in the IAA-free buffer, whereas it decreased immediately after incubation with IAA buffer. (2) Increase of PSIPT2 expression by IAA-depletion in nodal excised stem segments, in which PSIPT2 mRNA had been undetectable. The excised nodal segments from intact seedlings were incubated in MES buffer with or without IAA. Northern blot analyses demonstrated that PSIPT2 mRNA was induced in the IAA-free buffer, whereas it was not detected in the IAA buffer. In addition, PSIPT2 responds to the auxin level as well as the basipetal auxin flow in the stem, as indicated by the following two observations. (1) Induction of PSIPT2 mRNA is inhibited by the application of IAA in lanolin to the stump after decapitation. (2) PSIPT2 mRNA is induced by the application of TIBA in lanolin to the internode above the second node as well as by removal of the shoot apex. Auxin negatively regulates CK biosynthesis to control some plant developmental stages (Eklöf et al. 1997; Nordström et al. 2004), but there is little information on the molecular mechanisms occurring at the gene level. These above observations in pea plants indicate that auxin directly affects CK biosynthesis by regulating IPT expression, which is involved in the first step in CK biosynthesis.

$\mathrm{CK}$ is mainly synthesized in the root, which contains high levels of CK. In Arabidopsis, AtIPT1, AtIPT3, AtIPT5, and AtIPT7 transcripts are detected in the root (Miyawaki et al. 2004; Takei et al. 2004). In intact pea seedlings, the PsIPT2 expression level is very low in various organs, including root. Therefore, PSIPT2 may not contribute to CK biosynthesis in the root. PsIPT, however, may induce $\mathrm{CK}$ biosynthesis in the roots and other tissues to control various aspects of plant development. Members of the IPT family each have individual roles in controlling the complicated interactions between auxin and $\mathrm{CK}$, depending on plant development. 
Transgenic Arabidopsis seedlings containing the $\beta$-glucuronidase (GUS) reporter gene under control of the PSIPT2 promoter region $(2,025 \mathrm{bp})$ were generated to examine whether the depletion effect of auxin on PSIPT2 expression in pea is a common molecular mechanism in plants. The $G U S$ gene was expressed before treatment with IAA, but GUS mRNA was completely depleted by IAA treatment for $5 \mathrm{~h}$. This response of $G U S$ expression to auxin in transgenic Arabidopsis seedlings is similar to that of PSIPT2 expression in the pea stem, suggesting that plants, at least, pea and Arabidopsis, have similar molecular mechanisms in which auxin suppresses PsIPT2 expression (Tanaka et al. 2006).

\section{CK is locally biosynthesized in the nodal stem}

CK is mainly biosynthesized in roots, and xylem exudates contain high levels of CK. Based on the changes in the levels of $\mathrm{CK}$ in xylem exudates, it was thought that $\mathrm{CK}$ is transported from the root to promote axillary bud outgrowth (Letham 1994). In pea, CK levels in the stem and axillary buds increase $3 \mathrm{~h}$ after decapitation despite the fact that PSIPT2 is not expressed in growing axillary buds; therefore, CK is biosynthesized in the stem and transported into axillary buds to promote their outgrowth after decapitation.

Axillary bud outgrowth occurs in excised nodal stem segments without the root (Tamas et al. 1989). In pea, the amount of CK synthesized in the excised stem segments is the same as that in the stem with an intact root. Application of IAA in lanolin to the stump inhibits the increase in CK in the excised nodal stem segments. Together, these observations indicate that the $\mathrm{CK}$ in the stem is biosynthesized in the nodal stem and not transported from the root. The amount of $\mathrm{CK}$ locally biosynthesized de novo in the nodal stems is sufficient for promoting axillary bud outgrowth.

\section{PsPIN1 expression pattern follows the auxin flow in plants}

PIN1 has a very important role in polar auxin transport, because the Arabidopsis loss of PIN1 mutant has a very severe phenotype. The distribution of PIN-family proteins in Arabidopsis root follows the auxin flow (Blilou et al. 2005). PSPIN1 was isolated as a gene expressed specifically in the nodal stem of intact pea seedlings in a subtraction experiment. Although Gocal et al. had previously reported IAA levels in axillary buds of Phaseolus vulgaris (Gocal et al. 1991), more detailed measurements of IAA levels in axillary buds and nodal stems were made before and after decapitation in pea plants (Tanaka et al. unpublished data). The decrease in the IAA level in the stem after decapitation corresponded to the decrease in PsPIN1 expression in the stem. When auxin flow is recovered by de novo biosynthesis in outgrowing axillary buds, PsPIN1 expression in the stem also recovers. In axillary buds, the IAA level is low during dormancy, and increases in outgrowing axillary buds. In a similar manner, the PSPIN1 expression level in dormant axillary buds is low, whereas it increases in outgrowing axillary buds. These findings suggest that the PSPIN1 expression pattern follows the auxin flow in the stem, both before and after decapitation.

\section{The decrease in CK in the stem is also regulated by auxin through the regulation of cytokinin oxidase (CKX)}

Endogenous plant hormone levels are controlled by biosynthesis and metabolism. CKX is the only enzyme that inactivates $\mathrm{CK}$ by irreversibly degrading active CK (Jones and Schreiber 1997), and is a key factor in controlling the endogenous levels of active CK (Werner et al. 2001). CKX cDNA has been identified and characterized in various plants, such as maize, Arabidopsis, rice, Hordeum vulgare, Dendrobium hybrid, and Dendrobium huoshanense (Houba-Hérin et al. 1999; Morris et al. 1999). Two $C K X$ cDNAs (PsCKX1 and PsCKX2) from pea stem were isolated (Tanaka et al. unpublished data). PsCKX2 may contribute mainly to regulate $C K$ levels in the stem, because $P_{s} C K X 2$ transcript levels are very high compared with those of $P s C K X 1$ in the stem. PsCKX2 transcripts are expressed at a low level in the stem in intact plants. After decapitation, $P s C K X 2 \mathrm{mRNA}$ is undetectable from 3 to $9 \mathrm{~h}$ after decapitation, and then rapidly increases after $9 \mathrm{~h}$. The application of TIBA in lanolin to the stem causes a similar PsCKX2 expression pattern. These PsCKX2 expression patterns in the stem are opposite to the CK level patterns before and after decapitation, suggesting that PsCKX2 induces a decrease in CK levels in the stem.

In many cases, CKX activity and $C K X$ mRNA are promoted by $\mathrm{CK}$, indicating that $\mathrm{CK}$ levels are regulated by negative feedback through the regulation of CKX (Terrine and Laloue 1980; Brugière et al. 2003). On the other hand, auxin might induce some CKXs to regulate CK levels in various developmental processes in plants, because auxin induces the CKX activity during leaf development under canopy shade (Carabelli et al. 2007). In pea stem, PsCKX2 mRNA is strongly induced by auxin, indicating that auxin positively regulates $P s C K X 2$ expression (Tanaka et al. unpublished data). The decrease in CK in the stem $12 \mathrm{~h}$ after decapitation might be caused by both the suppression 
Fig. 1 A model of the interactions between auxin and $\mathrm{CK}$ in shoot branching through functions of PSIPT, PsCKX, and PsPIN1
(A) Shoot apex is intact

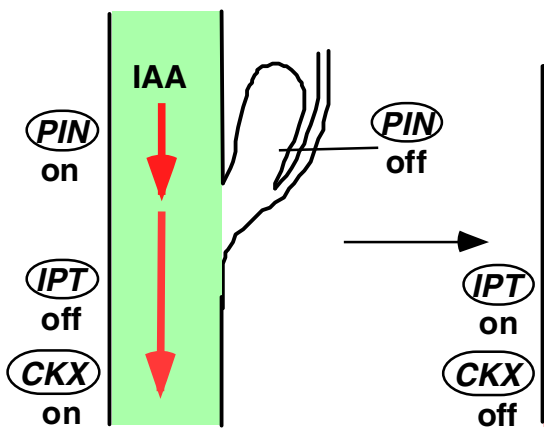

(B) After decapitation

(C) Axillary buds grow out of PSIPT2 expression and the induction of PsCKX2 in the stem. Auxin derived from the shoot apex might regulate CK levels in the stem by inducing CK degradation through the regulation of $C K X$ expression, as well as by the suppression of CK biosynthesis through the regulation of $I P T$ expression to control shoot branching. Plants seem to regulate plant hormone interactions to control developmental programs through complex crosstalk in which the hormones affect the expression of genes related to the biosynthesis as well as hormone metabolism.

\section{A model of interactions between auxin and $\mathrm{CK}$ in shoot branching through functions of PSIPT, PSCKX, and PSPIN1}

We propose the model of molecular mechanisms in shoot branching shown in Fig. 1. In an intact plant, basipetal auxin flow derived from the shoot apex represses PsIPT expression and maintains PSPIN1 expression in the stem. Consequently, axillary bud outgrowth does not occur. Once the shoot apex is decapitated, the auxin level in the stem decreases and repression of IPT expression is released. CK is then biosynthesized de novo in the stem and transported into dormant axillary buds to initiate their sustained outgrowth. After axillary bud outgrowth, de novo synthesized IAA derived from a new shoot apex flows to the stem, where it represses IPT expression and induces CKX to reduce the steady-state $\mathrm{CK}$ level in the stem.

\section{A third plant hormone involved in the control of shoot branching}

Recently, strigolactone, a group of terpenoid lactones, was identified as a third plant hormone involved in the control of shoot branching (Gomez-Roldan et al. 2008; Umehara et al. 2008). Gomez-Roldan et al. and Umehara et al. demonstrated that the levels of strigolactones are significantly reduced in the roots of enhanced shoot branching mutants (max/rms/dwarf). Moreover, the bushy phenotype of these mutants can be restored toward wild type when exogenous strigolactones are supplied. Strigolactones were previously reported to be communication chemicals in symbiotic arbuscular mycorrhizal fungi that facilitate soil nutrient uptake. Parasitic weeds, Striga and Orobanche, utilize strigolactone as a plant-derived signal to promote seed germination. To survive under nutrient-deficient conditions, plants might use strigolactones as signal molecules to suppress shoot branching and to communicate with symbiotic arbuscular mycorrhizal fungi. At present, the strigolactone biosynthesis and signal transduction pathways are not clear, and there is still much to be learned about the relationships between strigolactones, auxin, and CK with regard to the control of shoot branching. More information about the interactions between these three plant hormones will provide further insight into the molecular mechanisms of shoot branching.

Acknowledgments This work was supported in part by Grants-in Aid to H. M. from the Japan Ministry of Education, Sciences, Sports, and Culture (No. 19039015 and, 20061017). S. S.-S. has received Research Fellowships of the Japan Society for the Promotion of Science for Young Scientists (No. 19045188).

Open Access This article is distributed under the terms of the Creative Commons Attribution Noncommercial License which permits any noncommercial use, distribution, and reproduction in any medium, provided the original author(s) and source are credited.

\section{References}

Bangerth F (1994) Response of cytokinin concentration in the xylem exudate of bean (Phaseolus vulgaris L.) plants to decapitation and auxin treatment, and relationship to apical dominance. Planta 194:439-442. doi:10.1007/BF00197546

Beveridge CA, Ross JJ, Murfet IC (1994) Branching mutant $r m s-2$ in Pisum sativum. Plant Physiol 104:953-959

Blilou I, Xu J, Wildwater M, Willemsen V, Paponov I, Friml J, Heidstra R, Aida M, Palme K, Scheres B (2005) The PIN auxin efflux facilitator network controls growth and patterning in Arabidopsis roots. Nature 433:39-44. doi:10.1038/nature03184 
Brown BT, Foster C, Phillips JN, Rattigan BM (1979) The indirect role of 2, 4-D in the maintenance of apical dominance in decapitated sunflower seedlings (Helianthus annuus L.). Planta 146:475-480. doi:10.1007/BF00380863

Brugière N, Jiao S, Hantke S, Zinselmeier C, Roessler JA, Niu X, Jones RJ, Habben JE (2003) Cytokinin oxidase gene expression in maize is localized to the vasculature, and is induced by cytokinins, abscisic acid, and abiotic stress. Plant Physiol 132:1228-1240. doi:10.1104/pp.102.017707

Carabelli M, Possenti M, Sessa G, Ciolfi A, Sassi M, Morelli G, Ruberti I (2007) Canopy shade causes a rapid and transient arrest in leaf development through auxin-induced cytokinin oxidase activity. Genes Dev 21:1863-1868. doi:10.1101/gad.432607

Cline MG (1991) Apical dominance. Bot Rev 57:318-358. doi: 10.1007/BF02858771

Cline MG (1996) Exogenous auxin effects on lateral bud outgrowth in decapitated shoots. Ann Bot (Lond) 78:255-266. doi:10.1006/ anbo.1996.0119

Dun EA, Ferguson BJ, Beveridge CA (2006) Apical dominance and shoot branching. Divergent opinions or divergent mechanisms? Plant Physiol 142:812-819. doi:10.1104/pp.106.086868

Eklöf S, Åstot C, Blackwell J, Moritz T, Olsson O, Sandberg G (1997) Auxin-cytokinin interactions in wild-type and transgenic tobacco. Plant Cell Physiol 38:225-235

Gocal GFW, Pharis RP, Yeung EC, Pearce D (1991) Changes after decapitation in concentrations of indole-3-acetic acid and abscisic acid in the larger axillary bud of Phaseolus vulgaris L. cv tender green. Plant Physiol 95:344-350

Gomez-Roldan V, Fermas S, Brewer PB, Puech-Pagès V, Dun EA, Pillot J-P, Letisse F, Matusova R, Danoun S, Portais J-C, Bouwmeester H, Bécard G, Beveridge CA, Rameau C, Rochange SF (2008) Strigolactone inhibition of shoot branching. Nature 455:189-194. doi:10.1038/nature07271

Hall SM, Hillman JR (1975) Correlative inhibition of lateral bud growth in Phaseolus vulgaris L. Timing of bud growth following decapitation. Planta 123:137-143. doi:10.1007/BF00383862

Hopkins WG, Huner NPA (2004) Cytokinins are synthesized primarily in the root. In: Hopkins WG, Huner NP (eds) Introduction to plant physiology. Wiley, New York, pp 325-326

Houba-Hérin N, Pethe C, d'Alayer J, Laloue M (1999) Cytokinin oxidase from Zea mays: purification, cDNA cloning and expression in moss protoplasts. Plant $\mathrm{J}$ 17:615-626. doi: 10.1046/j.1365-313X.1999.00408.x

Ishikawa S, Maekawa M, Arite T, Onishi K, Takamure I, Kyozuka J (2005) Suppression of tiller bud activity in tillering dwarf mutants of rice. Plant Cell Physiol 46:79-86. doi:10.1093/pcp/pci022

Johnson CF, Morris DA (1989) Applicability of the chemiosmotic polar diffusion theory to the transport of indol-3yl-acetic acid in the intact pea (Pisum sativum L.). Planta 178:242-248. doi:10.1007/ BF00393200

Jones RJ, Schreiber BMN (1997) Role and function of cytokinin oxidase in plants. Plant Growth Regul 23:123-134. doi:10.1023/ A:1005913311266

Kakimoto T (2001) Identification of plant cytokinin biosynthetic enzymes as dimethylallyl diphosphate:ATP/ADP isopentenyltransferases. Plant Cell Physiol 42:677-685. doi:10.1093/ pcp/pce112

Kerstetter RA, Hake S (1997) Shoot meristem formation in vegetative development. Plant Cell 9:1001-1010. doi:10.1105/tpc.9.7.1001

Kitazawa D, Miyazawa Y, Fujii N, Hoshino A, Iida S, Nitasaka E, Takahashi H (2008) The gravity-regulated growth of axillary buds is mediated by a mechanism different from decapitationinduced release. Plant Cell Physiol 49:891-900. doi:10.1093/ $\mathrm{pcp} / \mathrm{pcn} 063$

Letham DS (1994) Cytokinin as phytohormones-sites of biosynthesis, translocation, and function of translocated cytokinin. In: Mok
DWS, Mok MC (eds) Cytokinin: chemistry, activity, and function. CRC Press, Florida, pp 57-80

Li CJ, Guevera E, Herrera J, Bangerth F (1995) Effect of apex excision and replacement by 1-naphthylacetic acid on cytokinin concentration and apical dominance in pea plants. Physiol Plant 94:465-469. doi:10.1111/j.1399-3054.1995.tb00955.x

Ljung K, Bhalerao RP, Sandberg G (2001) Sites and homeostatic control of auxin biosynthesis in Arabidopsis during vegetative growth. Plant J 28:465-474. doi:10.1046/j.1365-313X.2001. 01173.x

Lomax TL, Muday GK, Rubery PH (1995) Auxin transport. In: Davies PJ (ed) Plant hormones: physiology, biochemistry and molecular biology, 2nd edn. Kluwer Academic Publishers, Norwell, MA, pp 509-530

Malayer JC, Guard AT (1964) A comparative developmental study of the mutant side-shootless and normal tomato plants. Am J Bot 51:140-143. doi:10.2307/2440097

Marchant A, Kargul J, May ST, Muller P, Delbarre A, PerrotRechenmann C, Bennett MJ (1999) AUX1 regulates root gravitropism in Arabidopsis by facilitating auxin uptake within root apical tissues. EMBO J 18:2066-2073. doi:10.1093/emboj/ 18.8.2066

McIntyre GI, Damson EL (1988) Apical dominance in Phaseolus vulgaris. The triggering effect of shoot decapitation and leaf excision on growth of the lateral buds. Physiol Plant 74:607-614. doi:10.1111/j.1399-3054.1988.tb02025.x

Medford JI, Horgan R, El-Sawi Z, Klee HJ (1989) Alterations of endogenous cytokinins in transgenic plants using a chimeric isopentenyl transferase gene. Plant Cell 1:403-413

Miyawaki K, Matsumoto-Kitano M, Kakimoto T (2004) Expression of cytokinin biosynthetic isopentenyltransferase genes in Arabidopsis: tissue specificity and regulation by auxin, cytokinin, and nitrate. Plant J 37:128-138. doi:10.1046/j.1365-313X.2003. 01945.x

Morris RO, Bilyeu KD, Laskey JG, Cheikh NN (1999) Isolation of a gene encoding a glycosylated cytokinin oxidase from maize. Biochem Biophys Res Commun 255:328-333. doi:10.1006/bbrc. 1999.0199

Morris SE, Cox MCH, Ross JJ, Krisantini S, Beveridge CA (2005) Auxin dynamics after decapitation are not correlated with the initial growth of axillary buds. Plant Physiol 138:1665-1672. doi:10.1104/pp.104.058743

Murphy AS, Hoogner KR, Peer WA, Taiz L (2002) Identification, purification, and molecular cloning of $N$-1-naphthylphthalmic acid-binding plasma membrane-associated aminopeptidases from Arabidopsis. Plant Physiol 128:935-950. doi:10.1104/pp. 010519

Napoli C (1996) Highly branched phenotype of the petunia dad1-1 mutant is reversed by grafting. Plant Physiol 111:27-37

Nordström A, Tarkowski P, Tarkowska D, Norbaek R, Åstot C, Dolezal K, Sandberg G (2004) Auxin regulation of cytokinin biosynthesis in Arabidopsis thaliana: a factor of potential importance for auxin-cytokinin-regulated development. Proc Natl Acad Sci USA 101:8039-8044. doi:10.1073/pnas.0402 504101

Okada K, Ueda J, Komaki MK, Bell CJ, Shimura Y (1991) Requirement of the auxin polar transport system in early stages of Arabidopsis floral bud formation. Plant Cell 3:677-684

Ongaro V, Leyser O (2008) Hormonal control of shoot branching. J Exp Bot 59:67-74

Shimizu S, Mori H (1998) Analysis of cycles of dormancy and growth in pea axillary buds based on mRNA accumulation patterns of cell cycle-related genes. Plant Cell Physiol 39:255-262

Shimizu-Sato S, Mori H (2001) Control of outgrowth and dormancy in axillary buds. Plant Physiol 127:1405-1413. doi:10.1104/ pp.127.4.1405 
Snyder WE (1949) Some responses pf plants to 2, 3, 5-triiodo-benzoic acid. Plant Physiol 23:195-206

Stirnberg P, van de Sande K, Leyser HMO (2002) MAX1 and MAX2 control shoot lateral branching in Arabidopsis. Development 129:1131-1141

Takei K, Sakakibara H, Sugiyama T (2001) Identification of genes encoding adenylate isopentenyltransferase, a cytokinin biosynthesis enzyme, in Arabidopsis thaliana. J Biol Chem 276:2640526410. doi:10.1074/jbc.M102130200

Takei K, Ueda N, Aoki K, Kuromori T, Hirayama T, Shinozaki K, Yamaya T, Sakakibara H (2004) AtIPT3 is a key determinant of nitrate-dependent cytokinin biosynthesis in Arabidopsis. Plant Cell Physiol 45:1053-1062. doi:10.1093/pcp/pch119

Tamas IA, Schlossberg-Jacobs JL, Lim R, Friedman LB, Barone CC (1989) Effect of plant growth substances on the growth of axillary buds in cultured stem segments of Phaseolus vulgaris L. Plant Growth Regul 8:165-183. doi:10.1007/BF00024774

Tanaka M, Takei K, Kojima M, Sakakibara H, Mori H (2006) Auxin controls local cytokinin biosynthesis in the nodal stem in apical dominance. Plant J 45:1028-1036. doi:10.1111/j.1365-313X. 2006.02656.x

Terrine C, Laloue M (1980) Kinetics of $N^{6}-\left(\Delta^{2}\right.$-isopentenyl)adenosine degradation in tobacco cells: evidence of a regulatory mechanism under the control of cytokinins. Plant Physiol 65:1090-1095

Thimann KV, Skoog F (1934) On the inhibition of bud development and other functions of growth substance in Vicia faba. Proc $\mathrm{R}$ Soc Lond B Biol Sci 114:317-339

Turnbull CGN, Raymond MAA, Dodd IC, Morris SE (1997) Rapid increases in cytokinin concentration in lateral buds of chickpea (Cicer arietinum L.) during release of apical dominance. Planta 202:271-276. doi:10.1007/s004250050128

Umehara M, Hanada A, Yoshida S, Akiyama K, Arite T, TakedaKamiya N, Magome H, Kamiya Y, Shirasu K, Yoneyama K, Kyozuka J, Yamaguchi S (2008) Inhibition of shoot branching by new terpenoid plant hormones. Nature 455:195-200. doi: 10.1038/nature 07272

Werner T, Motyka V, Strnad M, Schmülling T (2001) Regulation of plant growth by cytokinin. Proc Natl Acad Sci USA 98:1048710492. doi: $10.1073 /$ pnas. 171304098

Wickson M, Thimann KV (1958) The antagonism of auxin and kinetin in apical dominance. Physiol Plant 49:304-314

Zubko E, Adams CJ, Macháèkova I, Malbeck J, Scollan C, Meyer P (2002) Activation tagging identifies a gene from Petunia hybrida responsible for the production of active cytokinins in plants. Plant J 29:797-808. doi:10.1046/j.1365-313X.2002.01256.x 